\title{
Machine-learned phase diagrams of generalized Kitaev honeycomb magnets
}

\author{
Nihal Rao $\odot,{ }^{1,2}$ Ke Liu (刘科 子竞) $\odot,{ }^{1,2, *}$ Marc Machaczek $\odot,{ }^{1,2}$ and Lode Pollet $\oplus^{1,2,3}$ \\ ${ }^{1}$ Arnold Sommerfeld Center for Theoretical Physics, University of Munich, Theresienstr. 37, 80333 München, Germany \\ ${ }^{2}$ Munich Center for Quantum Science and Technology (MCQST), Schellingstr. 4, 80799 München, Germany \\ ${ }^{3}$ Wilczek Quantum Center, School of Physics and Astronomy, Shanghai Jiao Tong University, Shanghai 200240, China
}

(Received 4 February 2021; revised 23 June 2021; accepted 9 August 2021; published 8 September 2021)

\begin{abstract}
We use a recently developed interpretable and unsupervised machine-learning method, the tensorial kernel support vector machine, to investigate the low-temperature classical phase diagram of a generalized HeisenbergKitaev- $\Gamma(J-K-\Gamma)$ model on a honeycomb lattice. Aside from reproducing phases reported by previous quantum and classical studies, our machine finds a hitherto missed nested zigzag-stripy order and establishes the robustness of a recently identified modulated $S_{3} \times Z_{3}$ phase, which emerges through the competition between the Kitaev and $\Gamma$ spin liquids, against Heisenberg interactions. The results imply that, in the restricted parameter space spanned by the three primary exchange interactions- $J, K$, and $\Gamma$, the representative Kitaev material $\alpha-\mathrm{RuCl}_{3}$ lies close to the boundaries of several phases, including a simple ferromagnet, the unconventional $S_{3} \times Z_{3}$, and nested zigzag-stripy magnets. A zigzag order is stabilized by a finite $\Gamma^{\prime}$ and/or $J_{3}$ term, whereas the four magnetic orders may compete in particular if $\Gamma^{\prime}$ is antiferromagnetic.
\end{abstract}

DOI: 10.1103/PhysRevResearch.3.033223

\section{INTRODUCTION}

Machine learning (ML) is quickly developing into a powerful tool in modern day physics research [1,2]. Successful applications in condensed-matter physics can be found in, for example, detecting phases and phase transitions [3-6], representing and solving quantum wave functions [7-12], analyzing experiments [13-15], searching new materials [16], and designing algorithms [17,18]. Recent developments of ML in strongly correlated condensed-matter physics are moving beyond benchmarking, and the ultimate goal is to provide toolboxes to tackle hard and open problems.

The Kitaev materials [19-21] are prime candidates for a challenging application of ML, hosting various disordered and unconventionally ordered phases. Experimentally, the bonddependent anisotropic interactions of the Kitaev honeycomb model [22] are realized through electron correlations and spinorbit coupling [23,24]. Representative compounds include $4 d$ and $5 d$ transition-metal-based Mott insulators $A_{2} \mathrm{IrO}_{3}(A=$ $\mathrm{Na}, \mathrm{Li}, \mathrm{K})$ and $\alpha-\mathrm{RuCl}_{3}$ [21,25-30]. In particular, the latter material has been proposed to host a field-induced quantum spin liquid as evidenced by the half-quantized thermal Hall effect under external magnetic field [31,32], while spectroscopic [33-35] and thermodynamic [36,37] measurements indicate a topologically trivial partially polarized phase. More recently, the cobaltate systems $\mathrm{Na}_{3} \mathrm{Co}_{2} \mathrm{SbO}_{6}$ and $\mathrm{Na}_{2} \mathrm{Co}_{2} \mathrm{TeO}_{6}$ [38-41]

\footnotetext{
*ke.liu@1mu.de

Published by the American Physical Society under the terms of the Creative Commons Attribution 4.0 International license. Further distribution of this work must maintain attribution to the author(s) and the published article's title, journal citation, and DOI.
}

and Cr-based pseudospin-3/2 systems $\mathrm{CrSiTe}_{3}$ and $\mathrm{CrGeTe}_{3}$ [42] were added to this family.

In the ideal case, one expects to find a compound that faithfully exhibits the physics of the Kitaev model. However, non-Kitaev terms, such as the Heisenberg exchange and the symmetric off-diagonal $\Gamma$ exchange, are permitted by the underlying cubic symmetry and ubiquitously exist in real Kitaev materials $[43,44]$. In addition, longer-range interactions and structural distortions can lead to further hopping channels $[35,45,46]$. These additional terms enrich the Kitaev physics [46-55] but also pose a significant challenge to the analysis because of the large parameter space and the emergence of complicated structures. Therefore, tools that can efficiently detect patterns and important information in data and construct the associated phase diagrams are called for.

In this work, we use our recently developed tensorialkernel support vector machine (TK-SVM) [56-58] to investigate the phase diagram of a generalized Heisenberg-Kitaev- $\Gamma$ model on a honeycomb lattice. This method is interpretable and unsupervised, equipped with a tensorial kernel and graph partitioning. The tensorial kernel detects both linear and high-order correlations, and the results can systematically be interpreted as meaningful physical quantities, such as order parameters [56] and emergent local constraints [58]. Moreover, in virtue of the graph partitioning module, phase diagrams can be constructed without supervision and prior knowledge.

In our previous investigation of the Kitaev magnets we applied TK-SVM to the classical $K-\Gamma$ model subject to a magnetic field [55]. There, our machine learned a rich global phase diagram, revealing, among others, two novel modulated $S_{3} \times Z_{3}$ phases, which originate from the competition between the Kitaev and $\Gamma$ spin liquids. This work explores the low-temperature classical phase diagram of the generic 
Heisenberg-Kitaev- $\Gamma(J-K-\Gamma)$ model as well as the effect of the $\Gamma^{\prime}$ and third-nearest-neighbor Heisenberg $\left(J_{3}\right)$ terms, which are subleading exchange terms commonly encountered in the class of Kitaev materials. From our findings it follows that in the parameter space spanned by $J, K$, and $\Gamma$, the representative Kitaev material $\alpha-\mathrm{RuCl}_{3}$ lies close to several competing phases, including a hitherto missed nested zigzag-stripy magnet, a previously identified $S_{3} \times Z_{3}$ magnet, a ferromagnet, and a possibly correlated paramagnet (Sec. III). Zigzag order can be stabilized by including a small $\Gamma^{\prime}$ and/or antiferromagnetic $J_{3}$ term. However, if the $\Gamma^{\prime}$ is also antiferromagnetic, this material resides in a region where these four magnetic orders strongly compete (Sec. IV). Our results constitute therefore one of the earliest examples of ML going beyond the state of the art in strongly correlated condensed matter physics.

This paper is organized as follows. In Sec. II, we define the generalized Heisenberg-Kitaev- $\Gamma$ model and specify the interested parameter regions. The machine-learned $J-K-\Gamma$ phase diagrams in the absence and presence of additional $J_{3}$ and $\Gamma^{\prime}$ terms are discussed and validated in Sec. III and Sec. IV, respectively. Section V discusses the implications of our results for representative Kitaev compounds. In Sec. VI we conclude and provide an outlook. In addition, a brief summary of TK-SVM and details about the training procedure and Monte Carlo simulations are provided in Appendixes.

\section{HONEYCOMB $J-K-\Gamma-\Gamma^{\prime}-J_{3}$ Model}

We study the generalized Heisenberg-Kitaev- $\Gamma$ model on a honeycomb lattice

$$
\begin{aligned}
H & =H_{J K \Gamma}+H_{\Gamma^{\prime}}+H_{J_{3}}, \\
& =\sum_{\langle i j\rangle_{\gamma}} \mathbf{S}_{i} \cdot \hat{\mathcal{J}}_{\gamma} \mathbf{S}_{j}+\sum_{(i j)} J_{3} \mathbf{S}_{i} \cdot \mathbf{S}_{j},
\end{aligned}
$$

where

$$
\begin{array}{r}
H_{J K \Gamma}=\sum_{\langle i j\rangle_{\gamma}}\left[J \mathbf{S}_{i} \cdot \mathbf{S}_{j}+K S_{i}^{\gamma} S_{j}^{\gamma}+\Gamma\left(S_{i}^{\alpha} S_{j}^{\beta}+S_{i}^{\beta} S_{j}^{\alpha}\right)\right], \\
H_{\Gamma^{\prime}}=\sum_{\langle i j\rangle_{\gamma}}\left[\Gamma^{\prime}\left(S_{i}^{\gamma} S_{j}^{\alpha}+S_{i}^{\gamma} S_{j}^{\beta}+S_{i}^{\alpha} S_{j}^{\gamma}+S_{i}^{\beta} S_{j}^{\gamma}\right)\right] .
\end{array}
$$

Here, $\gamma$ labels the three distinct nearest-neighbor (NN) bonds $\langle i j\rangle$ with mutually exclusive $\alpha, \beta, \gamma \in\{x, y, z\}$ as illustrated in Fig. $1 ; \hat{\mathcal{J}}_{\gamma}$ is a $3 \times 3$ matrix comprising all exchanges on a NN bond $\langle i j\rangle_{\gamma}$, and $(i j)$ denotes the third-NN bonds with a Heisenberg interaction $J_{3}$.

The Heisenberg-Kitaev- $\Gamma$ Hamiltonian Eq. (2) comprises generic $\mathrm{NN}$ exchanges allowed by the cubic symmetry $[23,43]$. Although the Kitaev $(K)$ term is of prime interest for realizing quantum Kitaev spin liquids, the Heisenberg $(J)$ and the symmetric off-diagonal $(\Gamma)$ exchanges ubiquitously exist and play a key role in the physics of realistic materials. The $\Gamma^{\prime}$ term is a secondary symmetric off-diagonal interaction and originates from a trigonal distortion of the octahedral environment of magnetic ions. A negative (positive) $\Gamma^{\prime}$ corresponds to trigonal compression (expansion) of the edgesharing oxygen or chlorine octahedra [45] while the inclusion of the $J_{3}$ term reflects the extension of $d$-electron wave functions. Although second-nearest-neighbor exchanges are also

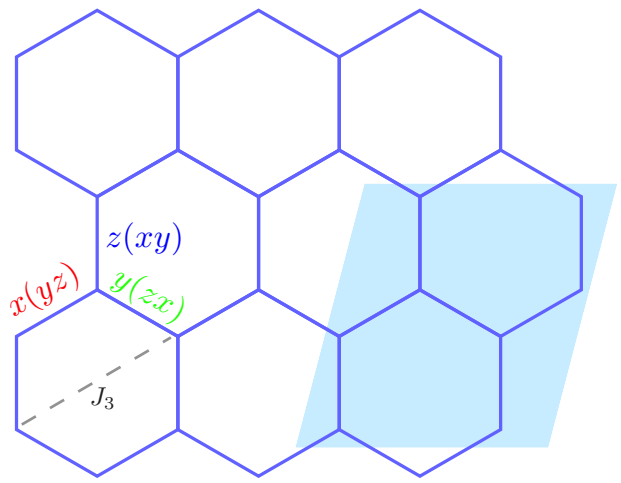

FIG. 1. A honeycomb lattice with anisotropic bonds $\gamma(\alpha \beta)$. The shaded region marks a symmetric cluster of $m \times m$ unit cells. A lattice with linear size $L$ is then partitioned into $\left(\frac{L}{m}\right)^{2}$ such clusters. Here, $m=2$ is shown for example. Larger clusters with $m=4,6,12$ are considered in training TK-SVMs.

possible, the third-neighbor exchanges are found to be more significant in representative Kitaev magnets, including the intensely studied compounds $\mathrm{Na}_{2} \mathrm{IrO}_{3}, \alpha-\mathrm{Li}_{2} \mathrm{IrO}_{3}, \alpha-\mathrm{RuCl}_{3}$ and the more recently (re)characterized cobalt-based compounds $\mathrm{Na}_{3} \mathrm{Co}_{2} \mathrm{SbO}_{6}$ and $\mathrm{Na}_{2} \mathrm{Co}_{2} \mathrm{TeO}_{6}[38,41]$. Aside from the potential microscopic origin, the $\Gamma^{\prime}$ and $J_{3}$ exchange terms are often introduced phenomenologically to stabilize magnetic orders observed in experiments [26,35,46,59], in particular the zigzag-type orders found in many two-dimensional Kitaev materials [19].

It is commonly expected that the primary physics in a Kitaev material is governed by the interactions in the $H_{J K}$ Г model, whose phase diagram for fixed $\Gamma^{\prime}$ and $J_{3}$ is the topic of the present work. Moreover, motivated by the microscopic models proposed for $\alpha-\mathrm{RuCl}_{3}[35,59], \mathrm{Na}_{3} \mathrm{Co}_{2} \mathrm{SbO}_{6}$ and $\mathrm{Na}_{2} \mathrm{Co}_{2} \mathrm{TeO}_{6}$ [41] (cf. Sec. IV), we focus on the parameter space with $K<0, \Gamma>0$ and a moderate range of ferromagnetic Heisenberg $(J)$ exchange terms.

Specifically, we parametrize the Kitaev and $\Gamma$ interactions as $K=\sin \theta, \Gamma=\cos \theta$, scan over $\theta \in\left[\frac{3}{2} \pi, 2 \pi\right]$ and restrict the Heisenberg interaction to $J \in[-0.3,0]$. We investigate slices of experimental relevance with $J_{3}=0,0.1$ and $\Gamma^{\prime}=$ $0, \pm 0.1$. In particular, considering a ferromagnetic as well as an antiferromagnetic $\Gamma^{\prime}$ covers both cases of its disputed sign in $\alpha-\mathrm{RuCl}_{3}$. Most of the previous studies considered a negative $\Gamma^{\prime}$ (trigonal compression) [60-63]. However, a recent work Ref. [35] advocates a positive $\Gamma^{\prime}$ (trigonal expansion) by stressing the electron-spin-resonance (ESR) [64] and terahertz $(\mathrm{THz})[34]$ experiments, and its critical magnetic fields $[54,65,66]$.

We treat spins as $O(3)$ vectors to gain training data for large system sizes, corresponding to the classical large- $S$ limit. We employ parallel-tempering Monte Carlo simulations with a heat bath algorithm and over relaxation to generate spin configurations and simulate large system sizes up to 10386 spins $(72 \times 72$ honeycomb unit cells), to accommodate potential competing orders. During the training procedure, $400(\theta, J)$ points are simulated for each fixed $\Gamma^{\prime}$ and $J_{3}$ slice, and in total 2,400 points are simulated. Training samples are collected at low temperature $T=10^{-3} \sqrt{K^{2}+\Gamma^{2}}$. Classification of these 
parameter points unravels the topology of the $J-K-\Gamma$ phase diagram for each of the six $\Gamma^{\prime}$ and $J_{3}$ combinations. Thereafter, we extract the physical order parameter of each phase from the learned TK-SVM decision functions. These order parameters are then measured in new simulations down to the temperature $T=10^{-4} \sqrt{K^{2}+\Gamma^{2}}$, in the most frustrated parameter regimes and passing through different phases and phase boundaries. The nature of the phases as well as the topologies of the machine-learned phase diagrams are thereby verified. See Appendix B for the setup of the sampling and training and Appendix $\mathrm{C}$ for details of the Monte Carlo simulations.

It turns out that the phase diagrams of the investigated parameter regions are dominated by various magnetic orders. This indicates that the classical phase diagrams may qualitatively, or even semiquantitatively, reflect those of finite spin- $S$ values. Indeed, we successfully reproduce the ferromagnetic, zigzag and $120^{\circ}$ orders previously observed in quantum and classical analysis $[35,43,46]$ and in addition find more phases.

\section{III. $J$-K-Г PHASE DIAGRAM}

We focus in this section on the machine-learned phase diagram for the pure Heisenberg-Kitaev- $\Gamma$ model and save the discussion on the effects of the $\Gamma^{\prime}$ and $J_{3}$ terms for Sec. IV. The $J-K-\Gamma$ phase diagram has previously been explored by several authors; see, for example, Refs. [30,43,46,47]. In the parameter regions with dominating Heisenberg and Kitaev exchanges, different methods give consistent results. The ferromagnetic, zigzag, antiferromagnetic, and stripy orders in the $J-K$ phase diagram $[24,67]$ extend to regions of finite $\Gamma$ $[43,46]$. The physics is, however, more subtle when the system is governed by competing Kitaev and $\Gamma$ interactions. In the parameter regime with $K<0, \Gamma \sim|K|$ and a small but finite ferromagnetic $J$ term, believed to be relevant for $\alpha-\mathrm{RuCl}_{3}$, a previous study based on a Luttinger-Tisza analysis suggests a zigzag order [43]. However, this order is not confirmed by the 24-site exact diagonalization (ED) carried out in the same work, and a more recent study [46] equipped with 32-site ED and cluster mean-field calculations shows that the physics depends on the size and shape of clusters.

Our machine finds that the phase diagram in the above parameter regime is quite rich, as shown in Fig. 2. In addition to reproduce the ferromagnetic and zigzag phase in the large $K$ and $\Gamma$ regions $[43,46,47]$ under a finite $J$, our machine also identifies a novel nested zigzag-stripy (ZZ-ST) phase and shows the extension of the $S_{3} \times Z_{3}$ phase. The $S_{3} \times Z_{3}$ phase results from the competition between the Kitaev and $\Gamma$ spin liquids and features a spin-orbit entangled modulation, with magnetic Bragg peaks at $\frac{2}{3} \mathbf{M}$ points [55].

The nested ZZ-ST order has not been reported in previous studies to the best of our knowledge. In this phase, whose representative ground-state configuration is illustrated in Fig. 3, spins can be divided into two groups, $\left\{\vec{S}_{A}, \vec{S}_{B}\right\}$. One set of spins, e.g., the $A$ spins in Fig. 3, form regular zigzag patterns with a doubled lattice constant while the other set of spins ( $B$ spins) form stripy patterns, intricately nested with the zigzag pattern of the $A$ spins. This nesting of orders enlarges the ground-state manifold: The global threefold rotation $\left(C_{3}\right)$ and spin-inversion symmetry $(S \rightarrow-S)$ of the (generalized)

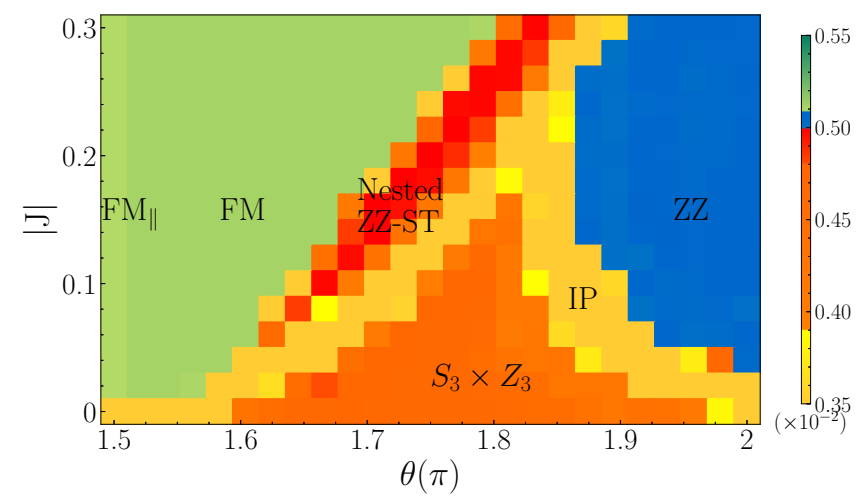

FIG. 2. Machine-learned $J-K-\Gamma$ phase diagram for parameters $J<0, K=\sin \theta<0, \quad \Gamma=\cos \theta>0$, at $T=10^{-3}$. Interactions and temperature are in units of $\sqrt{K^{2}+\Gamma^{2}}$. Each pixel represents a $(\theta, J)$ point with $\Delta \theta=\frac{1}{48} \pi$ and $\Delta J=0.02$; same for the phase diagrams below. A rank-1 TK-SVM with symmetric cluster of $12 \times 12$ lattice cells is used. The color represents the Fiedler entry value (FEV) for the corresponding $(\theta, J)$ point, and the choice of the color bar is guided by the histogram of FEVs (Appendix B). Parameter points in the same phase have the same or very close values. The blurry regions indicate phase boundaries and crossovers. The Kitaev and $\Gamma$ spin liquids reside at the corner of $(\theta, J)=\left(\frac{3}{2} \pi, 0\right)$ and $(2 \pi, 0)$, respectively, which are not distinguished from disordered IP regime as the rank-1 TK-SVM detects magnetic orders. FM: ferromagnetic, where $\mathrm{FM}_{\|}$indicates easy-axis states; nested ZZ-ST: nested zigzag-stripy; IP: incommensurate or (correlated) paramagnetic.

$J-K-\Gamma$ model trivially allows six ground states. This degeneracy is further doubled as the two sets of spins can be swapped, leading to twelve distinct ground states, which have all been observed in our Monte Carlo simulations. In addition, the

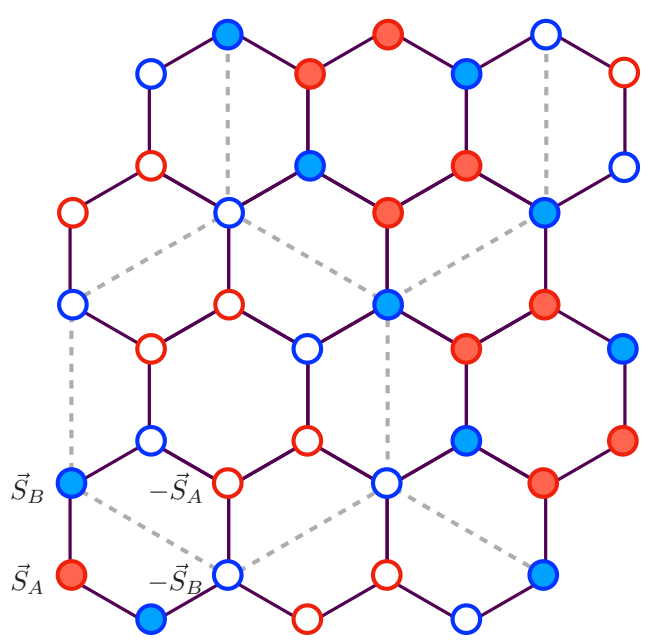

FIG. 3. A ground-state configuration of the nested zigzag-stripy order. The red $(A)$ and blue $(B)$ colors label two inequivalent reference spins, $\vec{S}_{A} \neq \vec{S}_{B}$. The filled (+) and empty (-) cycles indicate the sign of a spin. Here the $A$ spins ( $B$ spins) form zigzag (stripy) structures on a honeycomb lattice with a doubled lattice spacing. The dashed lines are a guide to the eye. 


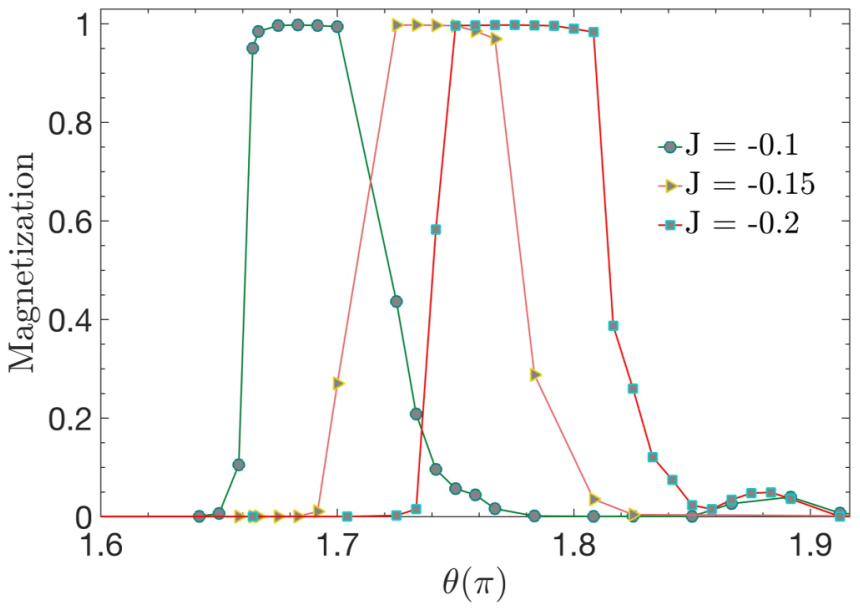

FIG. 4. Monte Carlo measurements of the nested zigzag-stripy magnetization at different $J$ 's, with $\Gamma^{\prime}=J_{3}=0, T=10^{-4}$. Consistent with the phase diagram Fig. 2 learned at $T=10^{-3}$, the nested zigzag-stripy order is preferred by larger $|J|$ and $\Gamma$.

robustness of the order is also confirmed in Fig. 4 by scanning $\theta$ at different $J$ values.

The formation of the $S_{3} \times Z_{3}$ and the nested ZZ-ST orders leads to an interesting evolution in spin structure factors (SSFs). As shown in Fig. 5 for a fixed $J=-0.1$, in the ferromagnetic phase at small $\Gamma$, the magnetic Bragg peak develops at the $\boldsymbol{\Gamma}$ point of the honeycomb Brillouin zone. Increasing the $\Gamma$ coupling results in the magnetic Bragg peaks moving outwards to the $\frac{1}{2} \mathbf{M}, \frac{2}{3} \mathbf{M}$, and $\mathbf{M}$ points, as the system passes the nested ZZ-ST, $S_{3} \times Z_{3}$, and zigzag orders, respectively.

These phases are nonetheless separated by broad crossover areas, marked as incommensurate or paramagnetic (IP) regimes, where our machine does not detect any clear magnetic ordering down to the temperature $T=10^{-3}$. Explicit measurements of the learned order parameters at a lower temperature $T=10^{-4}$ further show all magnetic moments are indeed remarkably fragile, as plotted in Fig. 6 with a fixed $J=-0.1$. Although with training data from a finitesize system and finite temperature, we cannot exclude lattice incommensuration and long-range orders at $T \rightarrow 0$ in these areas, our system size is considerably large and the absence of stable magnetic orders at such low temperatures is quite notable. One can expect quantum fluctuations will be enhanced in these areas as classical orders are suppressed, potentially hosting quantum paramagnets or spin liquids for finite spin- $S$ systems. The Kitaev and $\Gamma$ spin liquids are not distinguished from the disordered IP regimes in the phase diagram Fig. 2 as the rank-1 TK-SVM detects only magnetic correlations. However, as we studied in Ref. [55] for the $K-\Gamma$ model, while a classical $\Gamma$ SL are less robust against competing interactions, a classical KSL can thermally extend to a finite area.

\section{EFFECTS OF $\Gamma^{\prime}$ and $J_{3}$ term}

In modeling of Kitaev materials, the inclusion of the offdiagonal $\Gamma^{\prime}$ and third-neighbor Heisenberg $J_{3}$ exchange terms can have a phenomenological motivation or a microscopic origin, as discussed in Sec. II. In this section, we investigate their effects on the $J-K-\Gamma$ phase diagram.

\section{A. Finite $\Gamma^{\prime}$}

To disentangle their effects, we first study the case of $J_{3}=0$ and a finite $\Gamma^{\prime}$. The major consequence of adding a small ferromagnetic $\Gamma^{\prime}=-0.1$ is that the zigzag order in the $J-K-\Gamma$ phase diagram expands significantly and prevails over the phase diagram, as plotted in Fig. 7(a). In addition, a type of $120^{\circ}$ order [43,46] or antiferromagnetic $S_{3}$ order according to its order parameter structure [55], which originally lives in the $K>0, \Gamma>0$ region, is induced in the corner of large $\Gamma$ and small $J$. These results are consistent with the observations in Ref. [46] for the quantum spin-1/2 model.

Our machine finds more intricate physics for the $\Gamma^{\prime}=0.1$ case. As we show in Fig. 7(b), there are three stable magnetic phases. A ferromagnet and the nested ZZ-ST magnet dominate the parameter regions of small and large $\Gamma$, respectively, while the large- $\Gamma$ and small- $J$ limit accommodates an antiferromagnet. These phases are separated by broad crossovers. In particular, as shown in Fig. 8 along the $J=0$ line, in the regime between the nested and antiferromagnatic phase, no strong ordering is observed even down to the low-temperature $T=10^{-4}$. These regimes are hence also considered incommensurate or correlated paramagnetic (IP), similar as in the previous section for the $\Gamma^{\prime}=0$ case.

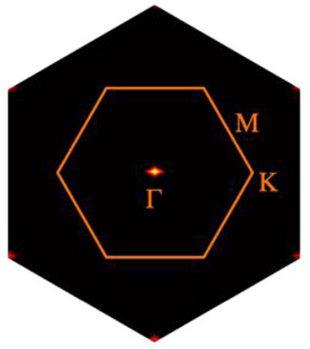

(a) FM

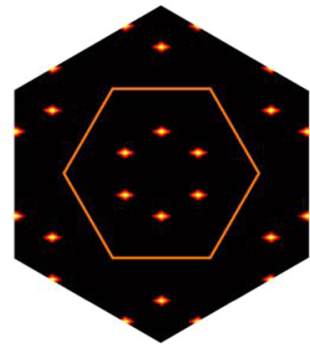

(b) ZZ-ST

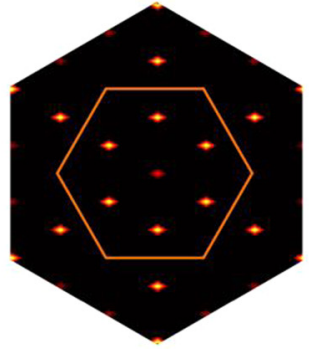

(c) $S_{3} \times Z_{3}$

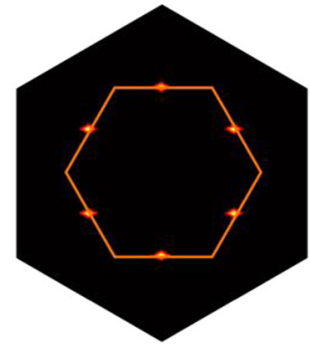

(d) $\mathrm{ZZ}$

FIG. 5. Evolution of the spin structure factor $S(\mathbf{q})$. The inner (outer) area denotes the first (second) honeycomb Brillouin zone; high symmetries points are indicated. Here $S(\mathbf{q})=\left\langle\frac{1}{2 L^{2}} \sum_{i j} \mathbf{S}_{i} \cdot \mathbf{S}_{j} e^{i \mathbf{q} \cdot\left(\mathbf{r}_{i}-\mathbf{r}_{j}\right)}\right\rangle$ is measured at $J=-0.1$ and $T=10^{-3}$. Upon increasing $\Gamma$, the magnetic Bragg peaks pass by the $\boldsymbol{\Gamma}(\mathrm{FM}), \frac{1}{2} \mathbf{M}$ (nested ZZ-ST), $\frac{2}{3} \mathbf{M}$ (modulated $S_{3} \times Z_{3}$ ), and $\mathbf{M}$ (zigzag) points. The length of the wave factors are stable within each phase. 


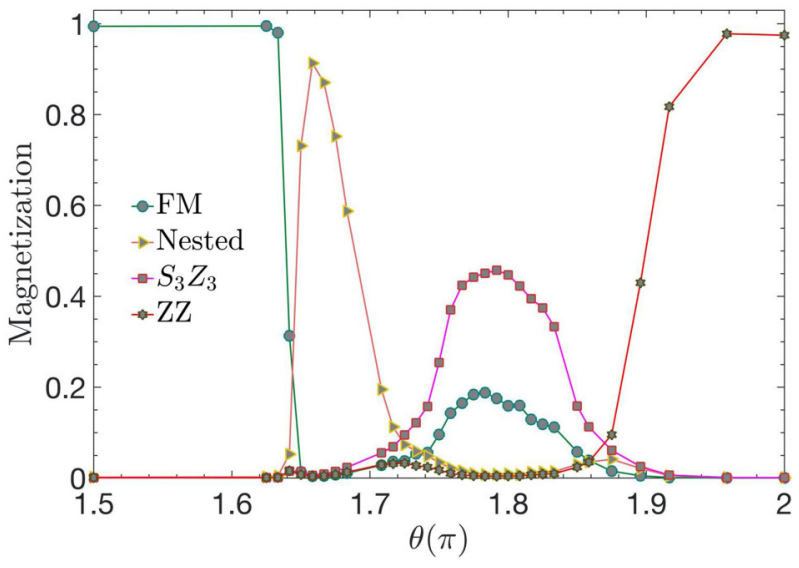

(a) $T=10^{-3}$

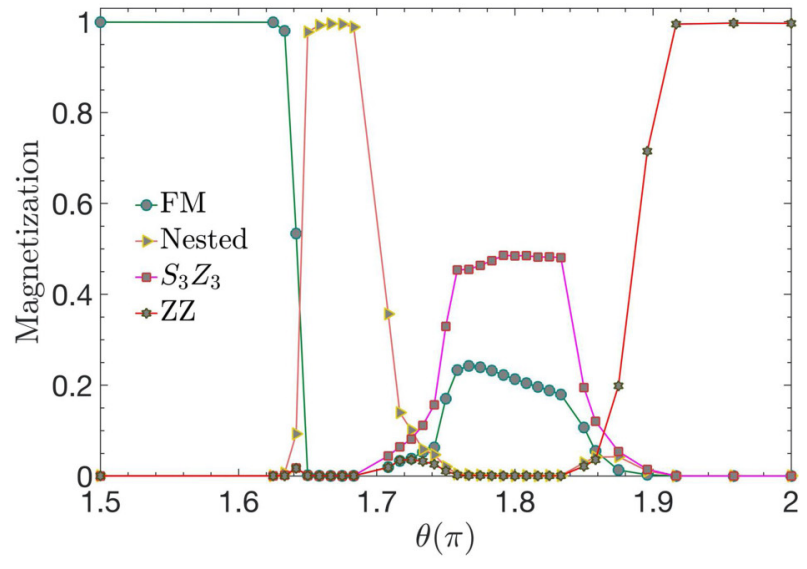

(b) $T=10^{-4}$

FIG. 6. Monte Carlo measurements of the magnetization at fixed $J=-0.1, \quad \Gamma^{\prime}=J_{3}=0$. Results for $T=10^{-3}$ and $T=10^{-4}$ are compared. The magnetic order of each phase in Fig. 2 is confirmed. The broad IP regions are narrower at very low temperature $T=10^{-4}$ but remain quite sizable, indicating that these regions are highly frustrated and the orders fragile.

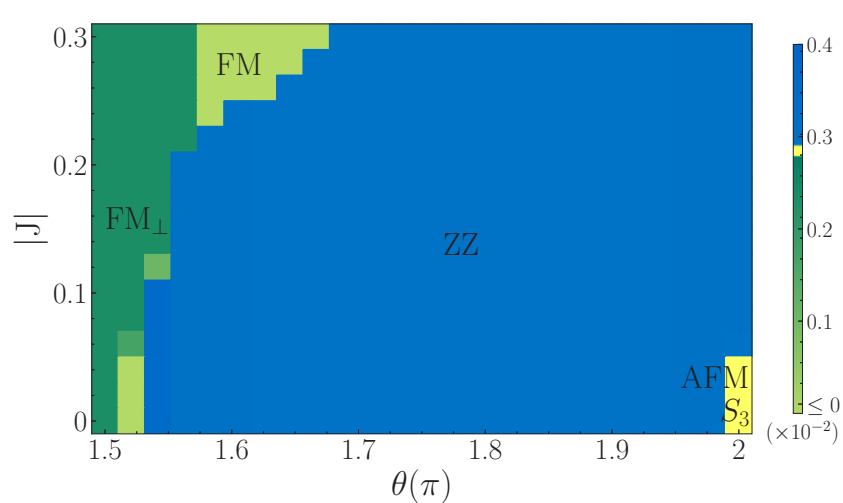

(a) $\Gamma^{\prime}=-0.1$

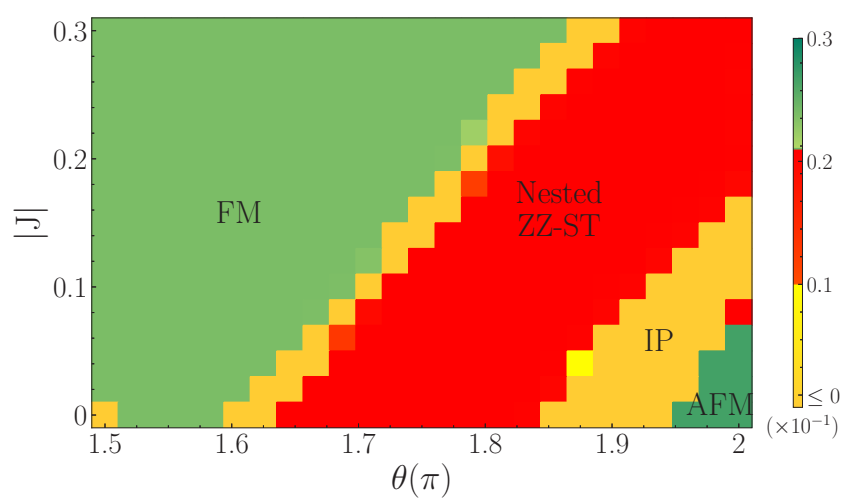

(b) $\Gamma^{\prime}=0.1$

FIG. 7. Machine-learned $J-K-\Gamma$ phase diagram, with $J_{3}=$ $0, \Gamma^{\prime}= \pm 0.1$ at $T=10^{-3}$. A zigzag phase prevails over the phase diagram when a ferromagnetic $\Gamma^{\prime}=-0.1$ is considered, while an antiferromagnetic $S_{3}$ order is stabilized in the large $\Gamma$ limit. All orders in (a) are unfrustrated. By contrast, in the case of an anti-ferromagnetic $\Gamma^{\prime}(\mathrm{b})$, the nested zigzag-stripy phase expands significantly, and there remains a highly frustrated IP region at larger $\Gamma$ values. (a) and (b) are learned with a symmetric $6 \times 6$ and $4 \times 4$ cluster, respectively. The resolution of $(\theta, J)$ points is same as in Fig. 2, namely, $\Delta \theta=\frac{1}{48} \pi$ and $\Delta J=0.02$. The corresponding Fiedler entries are color plotted in arbitrary unit, and their histograms are provided in Appendix B.

\section{B. Finite $J_{3}$ and $\Gamma^{\prime}$}

We now compile all the exchange interactions together. As shown in Fig. 9(a) and 9(b), the antiferromagetic $J_{3}$ exchange term strongly favors the zigzag order regardless of a vanishing or negative $\Gamma^{\prime}$, resulting in a simple topology to the phase diagram. As measured in Fig. 10, the zigzag moment prefers the directions of easy axes for $\Gamma^{\prime}=0$ and easy planes for $\Gamma^{\prime}=-0.1$ at small $\Gamma=0$, and evolves towards $\langle\overline{1} 11\rangle$ directions upon increasing $\Gamma$. An exception in the phase diagram is a small incommensurate or disordered area in the top left corner. This regime may be a remnant of a spiral order in the $O(3)$-symmetric $J_{1}-J_{3}$ honeycomb Heisenberg model $[68,69]$. It is present only in a narrow window around $J_{3} / J \sim-\frac{1}{3}$ along the $\Gamma=0$ line and becomes a trivial ferromagnet at larger $|J|$.

The combination of a positive $J_{3}$ and positive $\Gamma^{\prime}$ leads to a more complex topology, as shown in Fig. 9(c). While the zigzag phase still dominates the phase diagram, the ferromagnetic and the $S_{3} \times Z_{3}$ phase, relevant for the pure $J-K-\Gamma$ model

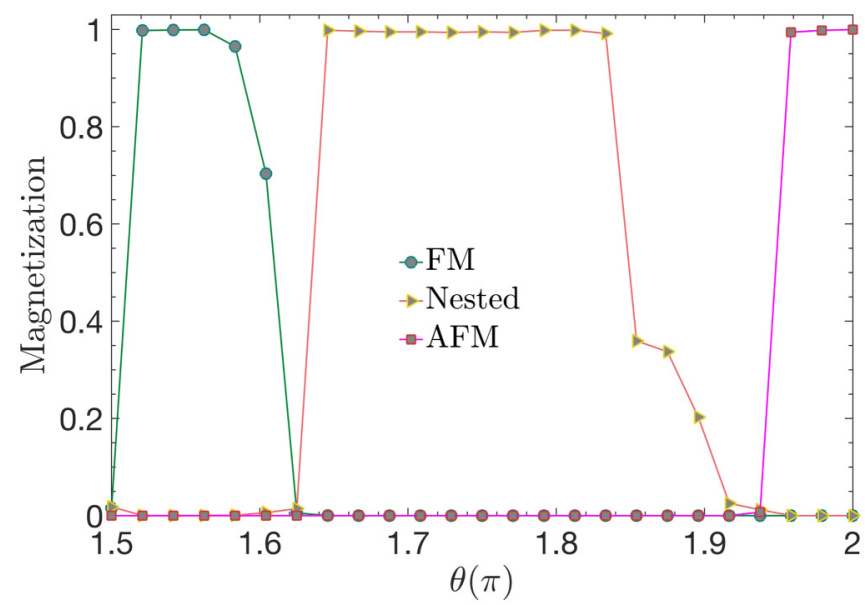

FIG. 8. Monte Carlo measurements of the magnetization with fixed $J=0, \quad \Gamma^{\prime}=0.1, J_{3}=0$, at $T=10^{-4}$. The wide window between the nested zigzag-stripy and antiferromagnetic orders corresponds to the IP regime in the phase diagram Fig. 7(b). 


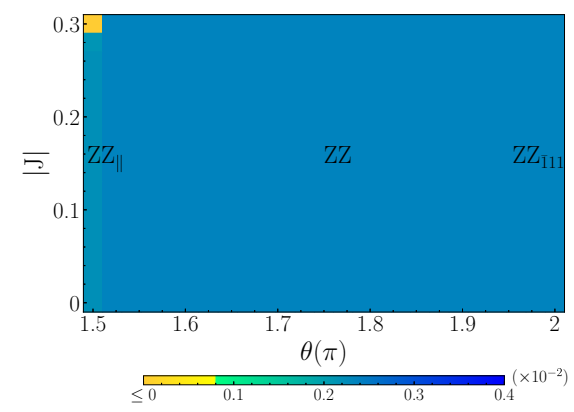

(a) $J_{3}=0.1, \Gamma^{\prime}=0$

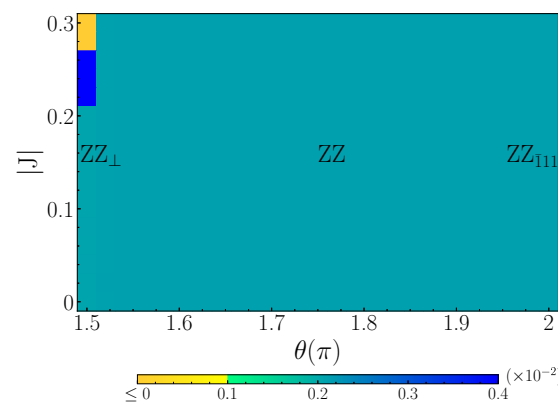

(b) $J_{3}=0.1, \Gamma^{\prime}=-0.1$

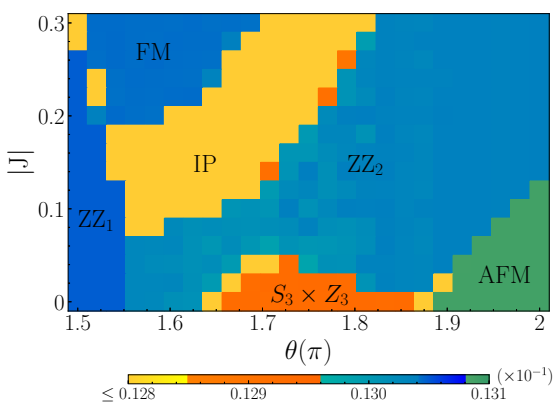

(c) $J_{3}=0.1, \Gamma^{\prime}=0.1$

FIG. 9. Machine-learned phase diagrams with $J_{3}=0.1, \Gamma^{\prime}=0, \pm 0.1$ at $T=10^{-3}$. The $J_{3}$ term universally prefers zigzag states. In cases of a vanishing and negative $\Gamma^{\prime}$, zigzag phases dominate the phase diagram, except for a narrow IP window at the $\Gamma=0$ limit with $J_{3} / J \sim-\frac{1}{3}$, which may be a remnant of a spiral order in the $J_{1}-J_{3}$ honeycomb model. Special zigzag states are marked in (a) and (b). With increasing $\Gamma$, the zigzag moment evolves from easy-axis $\left(\mathrm{ZZ}_{\|}\right)$or coplanar directions $\left(\mathrm{ZZ}_{\perp}\right)$ to $\langle\overline{1} 11\rangle$ directions $\left(\mathrm{ZZ}_{\overline{1} 11}\right)$. The system is more frustrated for antiferromagnetic $\Gamma^{\prime}$ (c). The zigzag phase closely competes with other orders and a broad IP region. $Z_{1}$ and $Z_{2}$ distinguish different zigzag configurations. (a) and (b) are learned with a symmetric $4 \times 4$ cluster, and (c) uses a $6 \times 6$ cluster. Colors reflect the value of Fiedler entries at each $(\theta, J)$ points, whose histograms are provided in Appendix B.

(Fig. 2), and the antiferromagnetic phase in the vanishing $J_{3}$ but positive $\Gamma^{\prime}$ case (Fig. 7), reappear. The nested ZZST order, which occupied a considerable area in the $J_{3}=0$ phase diagrams, is now taken over by an IP regime and a zigzag order. Clearly, a positive $\Gamma^{\prime}$ competes with $J_{3}$ and adds frustration.

\section{IMPLICATION TO MATERIALS}

We now apply the machine-learned phase diagrams to the representative parameter sets proposed for the compounds mentioned in Sec. II and reproduced in Table I. Following the parameters given in Ref. [41] based on inelastic neutron scattering (INS), the two cobaltate systems $\mathrm{Na}_{2} \mathrm{Co}_{2} \mathrm{TeO}_{6}$ and $\mathrm{Na}_{3} \mathrm{Co}_{2} \mathrm{SbO}_{6}$ both have a dominating ferromagnetic Kitaev exchange and a small antiferromagnetic $J_{3} \sim 0.1|K|$. They

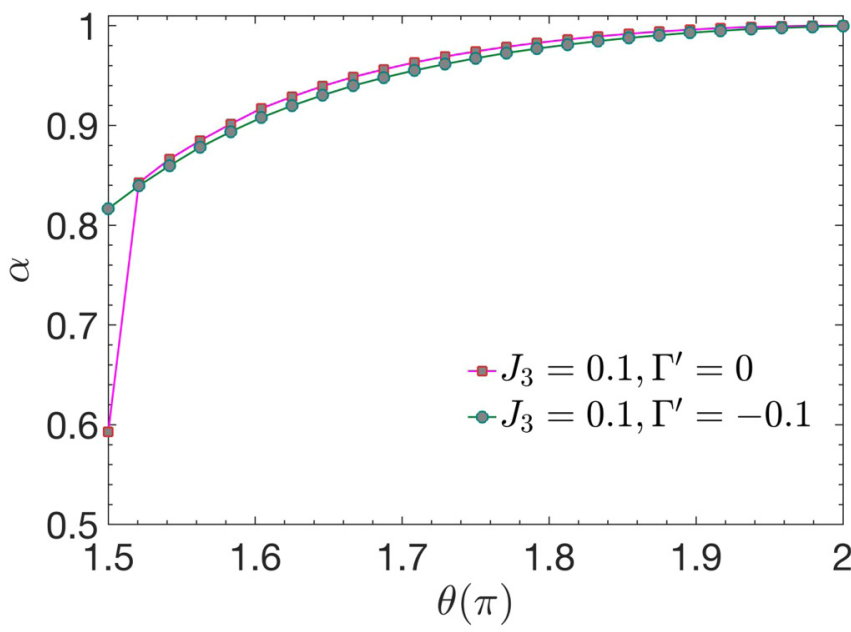

FIG. 10. Evolution of the zigzag moment $\left(\mathbf{m}_{\mathrm{ZZ}}\right)$ along the $J=$ -0.1 line, for $J_{3}=0.1, \Gamma^{\prime}=0,-0.1$, at $T=10^{-3}$. Spins prefer easy axes (easy planes) for the case of $\Gamma^{\prime}=0\left(\Gamma^{\prime}=-0.1\right)$ at small $\Gamma$, but evolves towards $\mathbf{n} \in\langle\overline{1} 11\rangle$ with increasing $\Gamma . \alpha=\left\langle\left|\mathbf{m}_{\mathrm{ZZ}} \cdot \mathbf{n}\right|\right\rangle$ measures the projection of the magnetic moment on directions of $\langle\overline{1} 11\rangle$, and $\langle$.$\rangle denotes an ensemble average.$ fall relatively deeper in the zigzag phase shown, respectively, in the phase diagrams of Figs. 9(a) and 9(b), in agreeement with the experimental result of Ref. [41].

The compound $\alpha-\mathrm{RuCl}_{3}$ also resides inside the zigzag phase, provided that the $\Gamma^{\prime}$ term is negligible, as suggested by the $a b$ initio calculation and the INS fit of Ref. [70], or is negative, as suggested by the DFT calculations of Refs. [60,61]. Nevertheless, if $\Gamma^{\prime}$ is antiferromagnetic, as was recently advocated in Ref. [35], it falls into the far more complex phase diagram Fig. 9(c). Consistent with the linear spin-wave analysis of Ref. [35], the zigzaglike magnet $\alpha-\mathrm{RuCl}_{3}$ will be then adjacent to an incommensurate or disordered regime.

However, it is interesting that, as indicated by Fig. 2, the projection of $\alpha-\mathrm{RuCl}_{3}$ on the $J-K-\Gamma$ subspace lies close to the frontier of several phases. Consider the commonly suggested range for the three major exchange interactions of this compound, $\Gamma \sim 0.5-1|K|(\theta \sim 1.65-1.75 \pi), J \sim-0.1|K|$ $[27,35,59-61,70]$. The relevant area in the $J-K-\Gamma$ phase diagram Fig. 2 encloses, or is close to the boundary of, the $S_{3} \times Z_{3}$, the nested ZZ-ST, a ferromagnetic phase and a broad paramagnetic regime. These phases may compete with the zigzag order stabilized by a finite $\Gamma^{\prime}$ and/or $J_{3}$ term, in particular if $\Gamma^{\prime}$ is antiferromagnetic.

\section{SUMMARY}

Kitaev materials are promising hosts of exotic phases and unconventionally ordered states of matter. Identifying the nature of those phases and constructing the associated phase diagrams is a daunting task. In this work we have utilized an interpretable and unsupervised machine-learning method, the tensorial kernel support vector machine (TK-SVM), to learn the phase diagram of a generalized Heisenberg-Kitaev- $\Gamma$ model on a honeycomb lattice.

Based on data from classical Monte Carlo simulations on large lattice size, the machine successfully reproduces the known magnetic orders as well as the incommensurate or paramagneticlike regimes reported in the previous quantum and classical studies. It also goes further by detecting new phases in the parameter regions relevant for the com- 
TABLE I. A selection of representative microscopic interactions (in meV) proposed for three Kitaev materials. A more complete collection of models suggested for $\alpha-\mathrm{RuCl}_{3}$ can be found in Refs. [59] and [35].

\begin{tabular}{|c|c|c|c|c|c|}
\hline & $J$ & $K$ & $\Gamma$ & $\Gamma^{\prime}$ & $J_{3}$ \\
\hline$\alpha-\mathrm{RuCl}_{3}[61]$ & -0.97 & -8.21 & 4.16 & -0.93 & \\
\hline$\alpha-\mathrm{RuCl}_{3}[60]$ & -1.67 & -6.67 & 6.6 & -0.87 & 2.8 \\
\hline$\alpha-\mathrm{RuCl}_{3}[70]$ & -0.5 & -5.0 & 2.5 & & 0.5 \\
\hline$\alpha-\mathrm{RuCl}_{3}[35]$ & {$[-4.1,-2.1]$} & {$[-11,-3.8]$} & {$[3.9,5.0]$} & {$[2.2,3.1]$} & {$[2.2,3.1]$} \\
\hline $\mathrm{Na}_{2} \mathrm{Co}_{2} \mathrm{TeO}_{6}[41]$ & $-0.1(8)$ & $-9.0(5)$ & $1.8(5)$ & $0.3(3)$ & $0.9(3)$ \\
\hline $\mathrm{Na}_{3} \mathrm{Co}_{2} \mathrm{SbO}_{6}$ [41] & $-2.0(5)$ & $-9.0(10)$ & $0.3(3)$ & $-0.8(2)$ & $0.8(2)$ \\
\hline
\end{tabular}

pounds $\alpha-\mathrm{RuCl}_{3}, \mathrm{Na}_{2} \mathrm{Co}_{2} \mathrm{TeO}_{6}$ and $\mathrm{Na}_{3} \mathrm{Co}_{2} \mathrm{SbO}_{6}$, including a nested zigzag-stripy phase and showing the extension of the modulated $S_{3} \times Z_{3}$ phase under finite Heisenberg interactions (Sec. III). In particular, the machine-learned phase diagrams suggest that, in the $J-K-\Gamma$ subspace, the actively studied compound $\alpha-\mathrm{RuCl}_{3}$ is situated near the boundary of several competing phases, including a simple ferromagnet, the more involved $S_{3} \times Z_{3}$ and nested zigzag-stripy magnets, and a possibly correlated paramagnet. The inclusion of further couplings such as $\Gamma^{\prime}$ and $J_{3}$ terms stabilizes zigzag order as known in the literature. However, if the $\Gamma^{\prime}$ exchange in this material is antiferromagnetic and sufficiently strong to compete with $J_{3}$, as recently put forward in Ref. [35], the proposed parameter set will be adjacent to an incommensurate or correlated paramagnetic regime, which may originate from the competition of the magnetic orders indicated above (Sec. IV).

To simulate large system sizes that unbiasedly accommodate different competing orders, we have treated spins as classical $O(3)$ vectors, corresponding to the large- $S$ limit of quantum spins. The fate of the novel orders identified by our machine against quantum fluctuations needs to be examined by future studies. Nevertheless, as strong symmetry-broken orders dominate, our phase diagrams can act as a useful reference for future quantum simulations. Moreover, by recognizing the unconventional orders and indicating the paramagneticlike regimes, our phase diagrams may also guide the understanding of existing Kitaev materials and the search for new materials.

We hope our study also stimulates future machine-learning applications in Kitaev materials. While such systems are motivated by material realizations $[23,24]$ of the Kitaev spin liquid [22], the presence of various non-Kitaev interactions appears to modify the physics expected from a pure Kitaev model considerably. Those interactions span a multidimensional parameter space, with accumulated evidence of complicated and rich phase diagrams. Machine-learning techniques are designed to discover important information and structure from high-dimensional complex data and can provide new toolboxes for investigating the physics of Kitaev materials and general frustrated magnets.

\section{ACKNOWLEDGMENTS}

N.R., K.L., M.M., and L.P. acknowledge support from FP7/ERC Consolidator Grant QSIMCORR, No. 771891, and the Deutsche Forschungsgemeinschaft (DFG, German Research Foundation) under Germany's Excellence Strategy -
EXC-2111 - 390814868. Our simulations make use of the $v$ SVM formulation [72], the LIBSVM library [73,74], and the ALPSCore library [75]. The TK-SVM library has been made openly available with documentation and examples [71]. The data used in this work are available upon request.

\section{APPENDIX A: TK-SVM}

Here we briefly review the TK-SVM method and refer to our previous work in Refs. [56-58,76] for a comprehensive introduction.

\section{Decision function}

In the language of TK-SVM, a phase classification problem is solved by learning a binary decision function

$$
d(\mathbf{x})=\sum_{\mu \nu} C_{\mu \nu} \phi_{\mu}(\mathbf{x}) \phi_{\nu}(\mathbf{x})-\rho .
$$

Here $\mathbf{x}=\left\{S_{i, a}\right\}$ represents a real-space snapshot of the system and serves as a training sample, with $i$ and $a$, respectively, labeling the lattice index and component of a spin. $\boldsymbol{\phi}(\mathbf{x})$ is a feature vector mapping $\mathbf{x}$ into degree- $n$ monomials,

$$
\phi^{(n)}: \mathbf{x} \rightarrow \boldsymbol{\phi}(\mathbf{x})=\left\{\phi_{\mu}\right\}=\left\{\left\langle S_{\alpha_{1}}^{a_{1}} S_{\alpha_{2}}^{a_{2}} \ldots S_{\alpha_{n}}^{a_{n}}\right\rangle_{\mathrm{cl}}\right\},
$$

where $\langle\ldots\rangle_{\mathrm{cl}}$ is a lattice average over finite clusters of $r$ spins, $\mu=\left\{\alpha_{n}, a_{n}\right\}$ denotes a collective index, $\alpha_{n}$ labels spins in a cluster, and the degree $n$ defines the rank of a TK-SVM kernel. The map $\phi^{(n)}$ is based on the observation that a symmetry-breaking order parameter or a local constraint for rotor degrees of freedom can be in general be represented by finite-rank tensors or polynomials [77-79]. With this map, the decision function probes both linear and higher-order correlators, including magnetic order, multipolar order, and ground-state constraints [56-58]. Moreover, this map can be combined with other machine-learning architectures, such as a principal component analysis (PCA). However, as elaborated in the thesis of Greitemann [76], it was found that TK-SVM has in general better performance and interpretability than TK-PCA. In a recent paper, Ref. [80], a nonlinear feature map with similar spirit was employed in a novel architecture of convolutional neural networks.

The coefficient matrix $C_{\mu \nu}$ in the decision function identifies important correlators that distinguish two data sets, from which order parameters can be extracted. It is defined as a weighted sum of support vectors,

$$
C_{\mu \nu}=\sum_{k} \lambda_{k} \phi_{\mu}\left(\mathbf{x}^{(k)}\right) \phi_{\nu}\left(\mathbf{x}^{(k)}\right),
$$




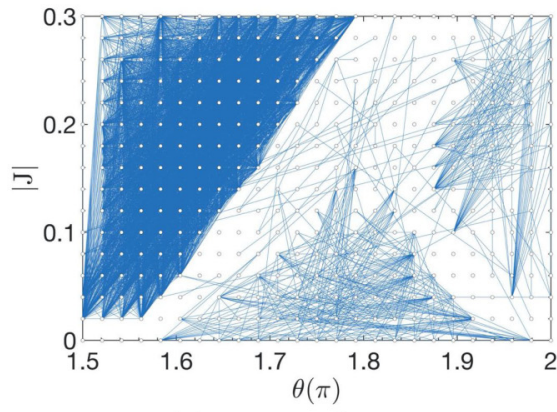

(a) $J_{3}=0, \Gamma^{\prime}=0$

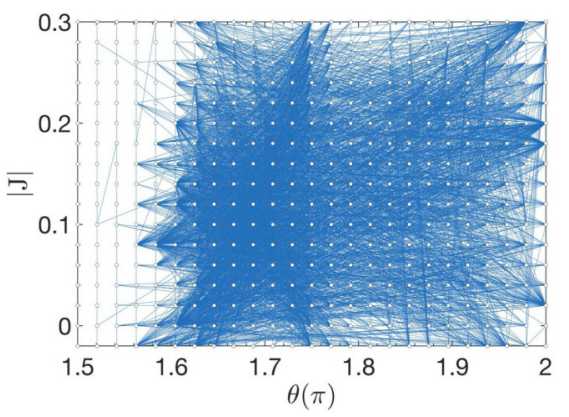

(d) $J_{3}=0.1, \Gamma^{\prime}=0$

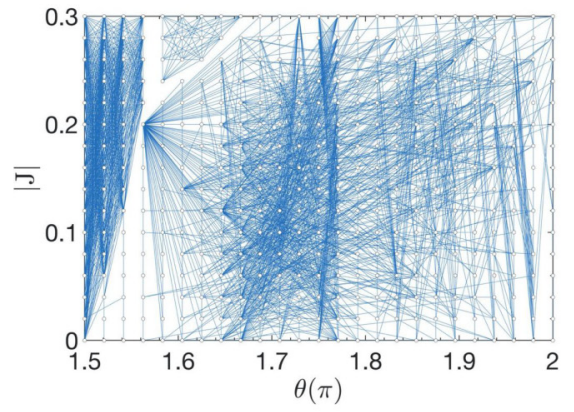

(b) $J_{3}=0, \Gamma^{\prime}=-0.1$

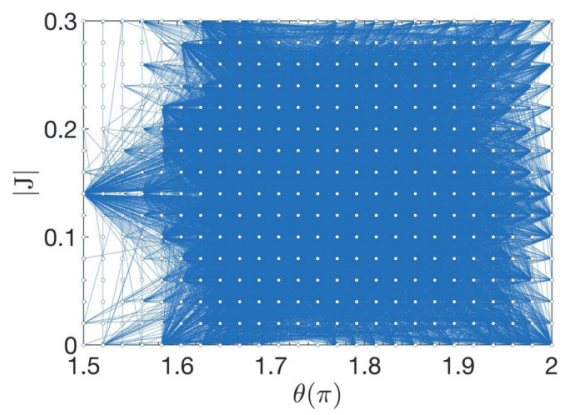

(e) $J_{3}=0.1, \Gamma^{\prime}=-0.1$

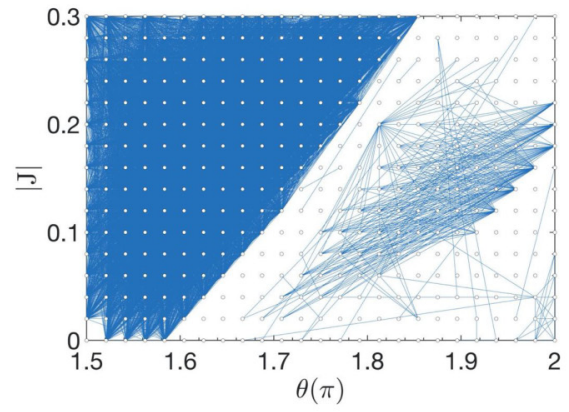

(c) $J_{3}=0, \Gamma^{\prime}=0.1$

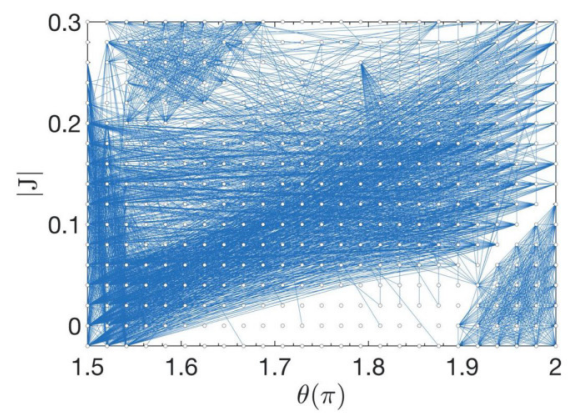

(f) $J_{3}=0.1, \Gamma^{\prime}=0.1$

FIG. 11. Graphs associated with the phase diagrams discussed in the main text. Each vertex (white circle) represents a $(\theta, J)$ point with fixed $J_{3}$ and $\Gamma^{\prime}$, from which training samples are collected. The edge (blue line) connecting two vertices is determined by the learned bias parameter $\rho$. Here $\rho_{c}=10^{4}$ is imposed in the weight function Eq. (A5). Each graph contains 400 vertices and 79,800 edges. Edge weights are suppressed in the figure for visualization purposes.

where $\lambda_{k}$ is a Lagrange multiplier and represents the weight of the $k$ th support vector.

The bias $\rho$ in Eq. (A1) is a normalization parameter in a standard SVM, but in TK-SVM it is endowed a physical implication to detect phase transitions and crossovers, or the absence thereof [58,77]. For two sample sets $p$ and $q$, it behaves as

$$
\left|\rho_{p q}\right| \begin{cases}\gg 1 & p, q \text { in the same phase } \\ \lesssim 1 & p, q \text { in different phases. }\end{cases}
$$

Although the sign of $\rho$ can indicate which data set is more disordered, its absolute value suffices to construct a phase diagram; cf. Ref. [58] for a comprehensive discussion.

The above binary classification is straightforwardly extended to a multiclassification problem over $M>1$ sets. SVM will then learn $M(M-1) / 2$ binary decision functions, comprising binary classifiers for each pair of sample sets [81].

\section{Graph partitioning}

Although the standard SVM is a supervised machinelearning method [82], the supervision can be skipped in the TK-SVM framework thanks to multiclassification and graph partitioning. A graph $G=(V, E)$ can be viewed as a pair of a vertex set $V$ and an edge set $E$ connecting vertices in $V$. Each vertex represents a phase point in the physical parameter space where we collect training data. For the $J-K-\Gamma$ phase diagram with fixed $\Gamma^{\prime}$ and $J_{3}$ couplings, these vertices are specified by the value of $\{\theta, J\}$. We work with weighted graphs. Namely, the edge linking two vertices $v_{p}, v_{q} \in V$ has a weight $w_{p q} \in$
$[0,1)$. Intuitively, if $v_{p}, v_{q}$ are in the same phase, they will be connected with a large $w_{p q}$; otherwise $w_{p q} \simeq 0$.

The weight of an edge is determined by the bias parameter, according to its behavior given in Eq (A4). The choice of the weighting function turns out not to be crucial. We adopt the normal Lorentzian weight distribution,

$$
w(\rho)=1-\frac{\rho_{c}^{2}}{(|\rho|-1)^{2}+\rho_{c}^{2}} \in[0,1),
$$

where $\rho_{c}$ is a hyperparameter setting a characteristic scale to quantify " $\gg 1$ ". The choice of $\rho_{c}$ is uncritical and does not rely on fine tuning, as edges connecting vertices in the same phase have always larger weights than those crossing a phase boundary. In practical use, we vary $\rho_{c}$ over several orders of magnitude to ensure the results are robust. In this work, $\rho_{c}=10^{2}, 10^{3}, 10^{4}$ are considered, and $\rho_{c}=10^{4}$ is chosen to construct the graphs in Fig. 11 and the subsequent phase diagrams. We refer to our previous works Refs. [58] and [83] for comparisons of results using different $\rho_{c}$ 's.

A graph of $M$ vertices and $M(M-1) / 2$ edges can be represented by a $M \times M$ Laplacian matrix

$$
\hat{L}=\hat{D}-\hat{A} \text {. }
$$

Here, $\hat{A}$ is a symmetric off-diagonal adjacency matrix with $A_{p q}=w_{p q}$ hosting the weights of the edges. $\hat{D}$ is a diagonal degree matrix where $D_{p p}=\sum_{p \neq q} w_{p q}$ denotes the degree of vertices.

We then utilize Fiedler's theory of spectral clustering to partition the graph $G[84,85]$, which is achieved by solving 


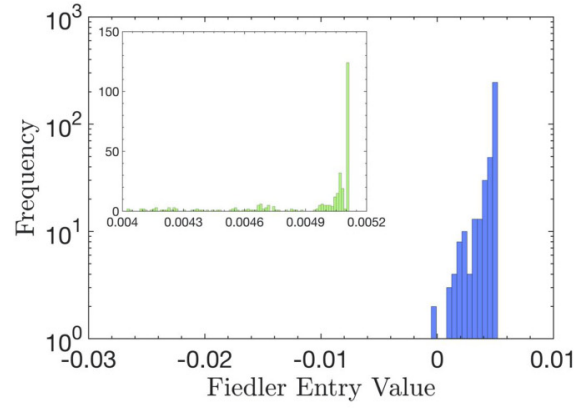

(a) $J_{3}=0, \Gamma^{\prime}=0$

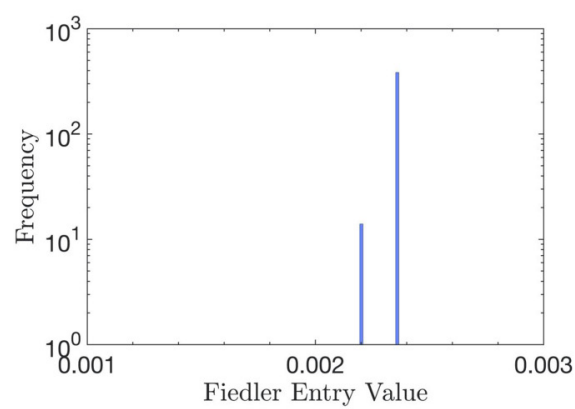

(d) $J_{3}=0.1, \Gamma^{\prime}=0$

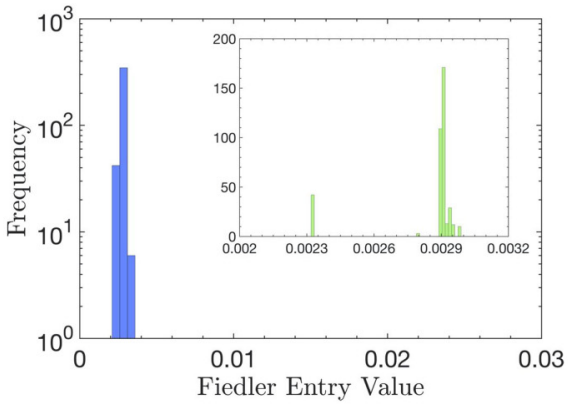

(b) $J_{3}=0, \Gamma^{\prime}=-0.1$

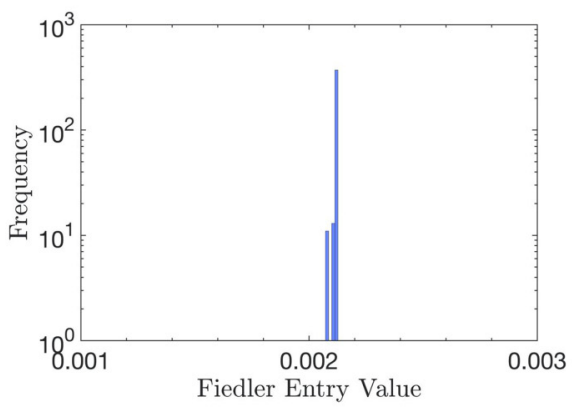

(e) $J_{3}=0.1, \Gamma^{\prime}=-0.1$

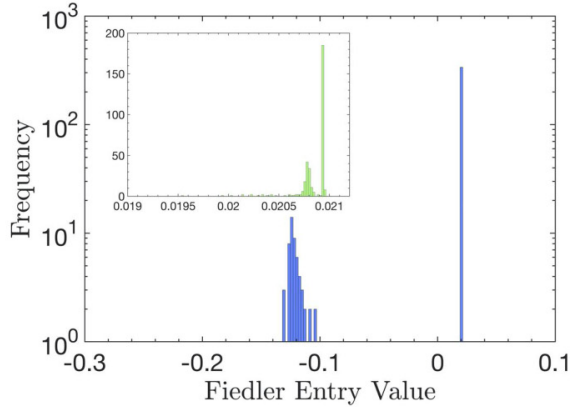

(c) $J_{3}=0, \Gamma^{\prime}=0.1$

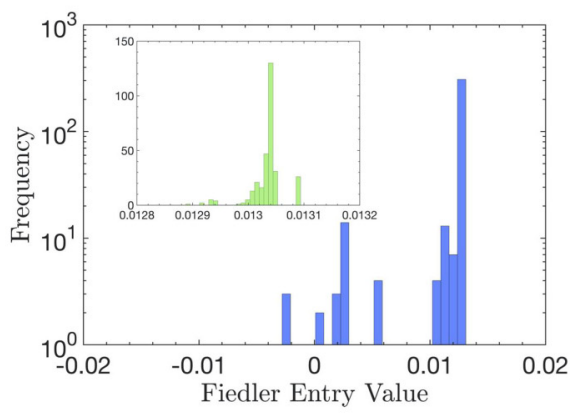

(f) $J_{3}=0.1, \Gamma^{\prime}=0.1$

FIG. 12. Histograms for the dominating Fiedler entries of the six graph partitioning problems. The main panels have a logarithmic scale on the vertical axis because the distribution spans several orders of magnitude. The insets show the main part of the distribution on a linear scale for easier comparison.

for the eigenvalues and eigenvectors of $\hat{L}$. The second smallest eigenvalue $\lambda_{2}$ reflects the algebraic connectivity of the graph, while the respective eigenvector $\mathbf{f}_{2}$ is known as the Fiedler vector. Entries of $\mathbf{f}_{2}$ have a one-to-one correspondence with vertices of the graph. Vertices (the physical parameter points) in the same subgraph will be assigned nearly identical Fiedler entries, while those in different subgraphs will be assigned contrasting values. The Fiedler vector can thereby effectively act as a phase diagram.

\section{APPENDIX B: SETUP OF THE SAMPLING AND LEARNING}

The parameters specified in Sec. II lead to six individual problems, depending on the value of $\Gamma^{\prime}$ and $J_{3}$. For fixed $\Gamma^{\prime}$ and $J_{3}, 400$ phase points are simulated in the $(J, \theta)$ subspace and 500 configurations are sampled at each point. These phase points distribute uniformly in the parameter range $J \in$ $[-0.3,0]$ and $\theta \in[1.5 \pi, 2 \pi]$, with $\Delta J=0.02, \Delta \theta=\frac{1}{48} \pi$. Such a protocol of sampling does not reflect a particular strategy but just represents a natural choice when exploring unknown phase diagrams.

We perform a TK-SVM multiclassification analysis on the sampled data with different clusters and ranks in the map $\phi^{(n)}$ in Eq. (A2). Each learning problem comprises 79800 binary decision functions, and a graph with 400 vertices and 79800 edges is constructed from the learned $\rho$ parameters, as visualized in Fig. 11. In all six cases, the phase diagrams can be mapped out with just rank-1 TK-SVMs, while a universal choice of the cluster is simply choosing a symmetric cluster with $m \times m$ honeycomb unit cells (see Fig. 1). We confirm the consistency of a phase diagram by checking the results against the ones found when using larger clusters with $m=4,6,12$ (32, 72, 288 spins).

The partitioning of these graphs leads to Fiedler vectors, which reveal the topology of the phase diagrams, and are color plotted in the main text. Figure 12 shows the histograms of the Fiedler vector entires. The pronounced peaks identify well-separated phases, and the flat regions indicate disordered regimes and crossovers between the phases.

After having determined the topology of the phase diagram, the coefficient matrix $C_{\mu \nu}$ is analyzed in order to extract the order parameter for distinct phases. In cases where no magnetic order is detected, we additionally perform a rank-2 TK-SVM analysis and identify a phase as a spin liquid if there is a stable ground-state constraint or as a correlated paramagnet or incommensurate phase if such a constraint is absent. The learned order parameters as well as the phase diagrams are validated by additional Monte Carlo simulations in Secs. III and IV.

\section{APPENDIX C: SIMULATION DETAILS}

We use parallel tempering (PT) in combination with the heat bath and over-relaxation algorithms to equilibrate the system. The distribution of temperatures are carefully chosen to ensure efficient iterations between different temperatures [86]. In the training stage, $N_{T}=64$ logarithmically equidistant temperatures between $T=0.5 \times 10^{-3}$ and 10 are used for the majority of the $(\theta, J)$ points, while $N_{T}=128$ temperatures are needed for a small subset of special parameter points. In the testing stage, $N_{T}=128$ temperatures in 
$\left[0.75 \times 10^{-4}, 10\right]$ are sufficient for most points measured in Figs. 4, 6, and 8, but $N_{T}=256$ temperatures are required for a few points.

We typically run $8 \times 10^{6}$ Monte Carlo (MC) sweeps for simulations using $N_{T}=64$ and $1.6 \times 10^{7}$ sweeps for those requiring more temperatures. Half of the total sweeps are considered as thermalization. In the training stage, 500 samples are collected from the second half of simulations. Namely, the sampling interval is 800 or 1600 MC sweeps. In the testing stage, new Monte Carlo simulations are performed to measure the learned order parameters (Figs. 4, 6, and 8), and several independent simulations are compared to confirm the ergodicity and good thermalization of our simulations. Alternatively, one could also measure the learned decision functions (without interpretation) as in applications of a standard SVM. As we established in Refs. [56,57], the TK-SVM decision function is essentially an encoder of physical order parameters. However, the goal of machine-learned phase diagrams is not only to assign each phase a different label but also to find suitable characterizations of the phases. Direct measurements of physical quantities are hence preferred if interpretability is guaranteed.
[1] G. Carleo, I. Cirac, K. Cranmer, L. Daudet, M. Schuld, N. Tishby, L. Vogt-Maranto, and L. Zdeborová, Machine learning and the physical sciences, Rev. Mod. Phys. 91, 045002 (2019).

[2] P. Mehta, M. Bukov, C.-H. Wang, A. G. R. Day, C. Richardson, C. K. Fisher, and D. J. Schwab, A high-bias, low-variance introduction to Machine Learning for physicists, Phys. Rep. 810, 1 (2019).

[3] L. Wang, Discovering phase transitions with unsupervised learning, Phys. Rev. B 94, 195105 (2016).

[4] P. Ponte and R. G. Melko, Kernel methods for interpretable machine learning of order parameters, Phys. Rev. B 96, 205146 (2017).

[5] J. Carrasquilla and R. G. Melko, Machine learning phases of matter, Nature Phys. 13, 431 (2017).

[6] L. Chen, X. Liang, and H. Zhai, The Bayesian committee approach for computational physics problems, arXiv:2011.06086.

[7] G. Carleo and M. Troyer, Solving the quantum many-body problem with artificial neural networks, Science 355, 602 (2017).

[8] J. Carrasquilla, Machine learning for quantum matter, Adv. Phys.: X 5, 1797528 (2020).

[9] D.-L. Deng, X. Li, and S. Das Sarma, Quantum entanglement in neural network states, Phys. Rev. X 7, 021021 (2017).

[10] J. Hermann, Z. Schätzle, and F. Noé, Deep-neural-network solution of the electronic Schrödinger equation, Nature Chem. 12, 891 (2020).

[11] D. Pfau, J. S. Spencer, A. G. D. G. Matthews, and W. M. C. Foulkes, $A b$ initio solution of the many-electron Schrödinger equation with deep neural networks, Phys. Rev. Research 2, 033429 (2020).

[12] T. Vieijra, C. Casert, J. Nys, W. De Neve, J. Haegeman, J. Ryckebusch, and F. Verstraete, Restricted Boltzmann Machines for Quantum States with Non-Abelian or Anyonic Symmetries, Phys. Rev. Lett. 124, 097201 (2020).

[13] Z. Nussinov, P. Ronhovde, D. Hu, S. Chakrabarty, B. Sun, N. A. Mauro, and K. K. Sahu, Inference of hidden structures in complex physical systems by multi-scale clustering, in Information Science for Materials Discovery and Design, edited by Turab Lookman, Francis J. Alexander, and Krishna Rajan (Springer International Publishing, Cham, 2016), pp. 115-138.

[14] Y. Zhang, A. Mesaros, K. Fujita, S. D. Edkins, M. H. Hamidian, K. Ch'ng, H. Eisaki, S. Uchida, J. C. Séamus Davis, E. Khatami, and E.-A. Kim, Machine learning in electronic-quantum-matter imaging experiments, Nature (London) 570, 484 (2019).
[15] A. Bohrdt, C. S. Chiu, G. Ji, M. Xu, D. Greif, M. Greiner, E. Demler, F. Grusdt, and M. Knap, Classifying snapshots of the doped Hubbard model with machine learning, Nature Phys. 15 921 (2019).

[16] J. Schmidt, M. R. G. Marques, S. Botti, and M. A. L. Marques, Recent advances and applications of machine learning in solidstate materials science, npj Comput. Mater. 5, 83 (2019).

[17] H.-J. Liao, J.-G. Liu, L. Wang, and T. Xiang, Differentiable Programming Tensor Networks, Phys. Rev. X 9, 031041 (2019).

[18] J. Liu, Y. Qi, Z. Y. Meng, and L. Fu, Self-learning monte carlo method, Phys. Rev. B 95, 041101(R) (2017).

[19] H. Takagi, T. Takayama, G. Jackeli, G. Khaliullin, and S. E. Nagler, Concept and realization of Kitaev quantum spin liquids, Nature Rev. Phys. 1, 264 (2019).

[20] L. Janssen and M. Vojta, Heisenberg-Kitaev physics in magnetic fields, J. Phys.: Condens. Matter 31, 423002 (2019).

[21] S. M Winter, A. A Tsirlin, M. Daghofer, J. van den Brink, Y. Singh, P. Gegenwart, and R. Valentí, Models and materials for generalized Kitaev magnetism, J. Phys.: Condens. Matter 29, 493002 (2017).

[22] A. Kitaev, Anyons in an exactly solved model and beyond, Ann. Phys. (NY) 321, 2 (2006).

[23] G. Jackeli and G. Khaliullin, Mott Insulators in the Strong SpinOrbit Coupling Limit: From Heisenberg to a Quantum Compass and Kitaev Models, Phys. Rev. Lett. 102, 017205 (2009).

[24] J. Chaloupka, G. Jackeli, and G. Khaliullin, Kitaev-Heisenberg Model on a Honeycomb Lattice: Possible Exotic Phases in Iridium Oxides $A_{2} \mathrm{IrO}_{3}$, Phys. Rev. Lett. 105, 027204 (2010).

[25] A. Banerjee, C. A. Bridges, J. Q. Yan, A. A. Aczel, L. Li, M. B. Stone, G. E. Granroth, M. D. Lumsden, Y. Yiu, J. Knolle, S. Bhattacharjee, D. L. Kovrizhin, R. Moessner, D. A. Tennant, D. G. Mandrus, and S. E. Nagler, Proximate Kitaev quantum spin liquid behaviour in a honeycomb magnet, Nature Mater. 15, 733 (2016).

[26] A. Banerjee, J. Yan, J. Knolle, C. A. Bridges, M. B. Stone, M. D. Lumsden, D. G. Mandrus, D. A. Tennant, R. Moessner, and S. E. Nagler, Neutron scattering in the proximate quantum spin liquid $\alpha-\mathrm{RuCl}_{3}$, Science 356, 1055 (2017).

[27] K. Ran, J. Wang, W. Wang, Z.-Y. Dong, X. Ren, S. Bao, S. Li, Z. Ma, Y. Gan, Y. Zhang, J. T. Park, G. Deng, S. Danilkin, S.-L. Yu, J.-X. Li, and J. Wen, Spin-Wave Excitations Evidencing the Kitaev Interaction in Single Crystalline $\alpha-\mathrm{RuCl}_{3}$, Phys. Rev. Lett. 118, 107203 (2017). 
[28] R. Yadav, N. A. Bogdanov, V. M. Katukuri, S. Nishimoto, J. van den Brink, and L. Hozoi, Kitaev exchange and field-induced quantum spin-liquid states in honeycomb $\alpha-\mathrm{RuCl}_{3}$, Sci. Rep. 6, 37925 (2016).

[29] R. Yadav, S. Nishimoto, M. Richter, J. van den Brink, and R. Ray, Large off-diagonal exchange couplings and spin liquid states in $C_{3}$-symmetric iridates, Phys. Rev. B 100, 144422 (2019).

[30] J. Chaloupka and G. Khaliullin, Hidden symmetries of the extended Kitaev-Heisenberg model: Implications for the honeycomb-lattice iridates $A_{2} \mathrm{IrO}_{3}$, Phys. Rev. B 92, 024413 (2015).

[31] Y. Kasahara, T. Ohnishi, Y. Mizukami, O. Tanaka, S. Ma, K. Sugii, N. Kurita, H. Tanaka, J. Nasu, Y. Motome, T. Shibauchi, and Y. Matsuda, Majorana quantization and half-integer thermal quantum hall effect in a Kitaev spin liquid, Nature (London) 559, 227 (2018).

[32] T. Yokoi, S. Ma, Y. Kasahara, S. Kasahara, T. Shibauchi, N. Kurita, H. Tanaka, J. Nasu, Y. Motome, C. Hickey, S. Trebst, and Y. Matsuda, Half-integer quantized anomalous thermal Hall effect in the Kitaev material $\alpha-\mathrm{RuCl}_{3}$, Science 373, 568 (2021).

[33] A. N. Ponomaryov, L. Zviagina, J. Wosnitza, P. Lampen-Kelley, A. Banerjee, J.-Q. Yan, C. A. Bridges, D. G. Mandrus, S. E. Nagler, and S. A. Zvyagin, Nature of Magnetic Excitations in the High-Field Phase of $\alpha-\mathrm{RuCl}_{3}$, Phys. Rev. Lett. 125, 037202 (2020).

[34] A. Sahasrabudhe, D. A. S. Kaib, S. Reschke, R. German, T. C. Koethe, J. Buhot, D. Kamenskyi, C. Hickey, P. Becker, V. Tsurkan, A. Loidl, S. H. Do, K. Y. Choi, M. Grüninger, S. M. Winter, Z. Wang, R. Valentí, and P. H. M. van Loosdrecht, High-field quantum disordered state in $\alpha-\mathrm{RuCl}_{3}$ : Spin flips, bound states, and multiparticle continuum, Phys. Rev. B 101, 140410(R) (2020).

[35] P. A. Maksimov and A. L. Chernyshev, Rethinking $\alpha-\mathrm{RuCl}_{3}$, Phys. Rev. Research 2, 033011 (2020).

[36] S. Bachus, D. A. S. Kaib, Y. Tokiwa, A. Jesche, V. Tsurkan, A. Loidl, S. M. Winter, A. A. Tsirlin, R. Valentí, and P. Gegenwart, Thermodynamic Perspective on Field-Induced Behavior of $\alpha-\mathrm{RuCl}_{3}$, Phys. Rev. Lett. 125, 097203 (2020).

[37] S. Bachus, D. A. S. Kaib, A. Jesche, V. Tsurkan, A. Loidl, S. M. Winter, A. A. Tsirlin, R. Valentí, and P. Gegenwart, Angle-dependent thermodynamics of $\alpha-\mathrm{RuCl}_{3}$, Phys. Rev. B 103, 054440 (2021).

[38] L. Viciu, Q. Huang, E. Morosan, H. W. Zandbergen, N. I. Greenbaum, T. McQueen, and R. J. Cava, Structure and basic magnetic properties of the honeycomb lattice compounds $\mathrm{Na}_{2} \mathrm{Co}_{2} \mathrm{TeO}_{6}$ and $\mathrm{Na}_{3} \mathrm{Co}_{2} \mathrm{SbO}_{6}$, J. Solid State Chem. France 180, 1060 (2007).

[39] H. Liu and G. Khaliullin, Pseudospin exchange interactions in $d^{7}$ cobalt compounds: Possible realization of the Kitaev model, Phys. Rev. B 97, 014407 (2018).

[40] H. Liu, J. Chaloupka, and G. Khaliullin, Kitaev Spin Liquid in $3 d$ Transition Metal Compounds, Phys. Rev. Lett. 125, 047201 (2020).

[41] M. Songvilay, J. Robert, S. Petit, J. A. Rodriguez-Rivera, W. D. Ratcliff, F. Damay, V. Balédent, M. Jiménez-Ruiz, P. Lejay, E. Pachoud, A. Hadj-Azzem, V. Simonet, and C. Stock, Kitaev interactions in Co honeycomb antiferromagnets $\mathrm{Na}_{3} \mathrm{Co}_{2} \mathrm{SbO}_{6}$ and $\mathrm{Na}_{2} \mathrm{Co}_{2} \mathrm{TeO}_{6}$, Phys. Rev. B 102, 224429 (2020).
[42] C. Xu, J. Feng, M. Kawamura, Y. Yamaji, Y. Nahas, S. Prokhorenko, Y. Qi, H. Xiang, and L. Bellaiche, Possible Kitaev Quantum Spin Liquid State in 2D Materials with $S=3 / 2$, Phys. Rev. Lett. 124, 087205 (2020).

[43] J. G. Rau, E. K.-H. Lee, and H.-Y. Kee, Generic Spin Model for the Honeycomb Iridates Beyond the Kitaev Limit, Phys. Rev. Lett. 112, 077204 (2014).

[44] J. G. Rau, E. K.-H. Lee, and H.-Y. Kee, Spin-orbit physics giving rise to novel phases in correlated systems: Iridates and related materials, Ann. Rev. Condens. Matter Phys. 7, 195 (2016).

[45] J. G. Rau and H.-Y. Kee, Trigonal distortion in the honeycomb iridates: Proximity of zigzag and spiral phases in $\mathrm{Na}_{2} \mathrm{IrO}_{3}$, arXiv:1408.4811.

[46] J. Rusnačko, D. Gotfryd, and J. Chaloupka, Kitaev-like honeycomb magnets: Global phase behavior and emergent effective models, Phys. Rev. B 99, 064425 (2019).

[47] J. Wang, B. Normand, and Z.-X. Liu, One Proximate Kitaev Spin Liquid in the $K-J-\Gamma$ Model on the Honeycomb Lattice, Phys. Rev. Lett. 123, 197201 (2019).

[48] M. Gohlke, G. Wachtel, Y. Yamaji, F. Pollmann, and Y. B. Kim, Quantum spin liquid signatures in Kitaev-like frustrated magnets, Phys. Rev. B 97, 075126 (2018).

[49] M. Gohlke, L. E. Chern, H.-Y. Kee, and Y. B. Kim, Emergence of nematic paramagnet via quantum order-by-disorder and pseudo-goldstone modes in kitaev magnets, Phys. Rev. Research 2, 043023 (2020).

[50] L. E. Chern, R. Kaneko, H.-Y. Lee, and Y. B. Kim, Magnetic field induced competing phases in spin-orbital entangled Kitaev magnets, Phys. Rev. Research 2, 013014 (2020).

[51] H.-Y. Lee, R. Kaneko, L. E. Chern, T. Okubo, Y. Yamaji, N. Kawashima, and Y. B. Kim, Magnetic field induced quantum phases in a tensor network study of Kitaev magnets, Nature Commun. 11, 1639 (2020).

[52] Y.-F. Jiang, T. P. Devereaux, and H.-C. Jiang, Field-induced quantum spin liquid in the Kitaev-Heisenberg model and its relation to $\alpha-\mathrm{RuCl}_{3}$, Phys. Rev. B 100, 165123 (2019).

[53] J. S. Gordon, A. Catuneanu, E. S. Sørensen, and H.-Y. Kee, Theory of the field-revealed Kitaev spin liquid, Nature Commun. 10, 2470 (2019).

[54] P. Lampen-Kelley, L. Janssen, E. C. Andrade, S. Rachel, J.-Q. Yan, C. Balz, D. G. Mandrus, S. E. Nagler, and M. Vojta, Fieldinduced intermediate phase in $\alpha-\mathrm{RuCl}_{3}$ : Non-coplanar order, phase diagram, and proximate spin liquid, arXiv:1807.06192.

[55] K. Liu, N. Sadoune, N. Rao, J. Greitemann, and L. Pollet, Revealing the phase diagram of kitaev materials by machine learning: Cooperation and competition between spin liquids, Phys. Rev. Research 3, 023016 (2021).

[56] J. Greitemann, K. Liu, and L. Pollet, Probing hidden spin order with interpretable machine learning, Phys. Rev. B 99, 060404(R) (2019).

[57] K. Liu, J. Greitemann, and L. Pollet, Learning multiple order parameters with interpretable machines, Phys. Rev. B 99, 104410 (2019).

[58] J. Greitemann, K. Liu, L. D. C. Jaubert, H. Yan, N. Shannon, and L. Pollet, Identification of emergent constraints and hidden order in frustrated magnets using tensorial kernel methods of machine learning, Phys. Rev. B 100, 174408 (2019). 
[59] P. Laurell and S. Okamoto, Dynamical and thermal magnetic properties of the Kitaev spin liquid candidate $\alpha-\mathrm{RuCl}_{3}, \mathrm{npj}$ Quantum Mater. 5, 2 (2020).

[60] H.-S. Kim and H.-Y. Kee, Crystal structure and magnetism in $\alpha-\mathrm{RuCl}_{3}$ : An ab initio study, Phys. Rev. B 93, 155143 (2016).

[61] S. M. Winter, Y. Li, H. O. Jeschke, and R. Valentí, Challenges in design of Kitaev materials: Magnetic interactions from competing energy scales, Phys. Rev. B 93, 214431 (2016).

[62] C. Eichstaedt, Y. Zhang, P. Laurell, S. Okamoto, A. G. Eguiluz, and T. Berlijn, Deriving models for the Kitaev spin-liquid candidate material $\alpha-\mathrm{RuCl}_{3}$ from first principles, Phys. Rev. B 100, 075110 (2019).

[63] J. A. Sears, L. E. Chern, S. Kim, P. J. Bereciartua, S. Francoual, Y. B. Kim, and Y.-J. Kim, Ferromagnetic Kitaev interaction and the origin of large magnetic anisotropy in $\alpha-\mathrm{RuCl}_{3}$, Nature Phys. 16, 837 (2020).

[64] A. N. Ponomaryov, E. Schulze, J. Wosnitza, P. Lampen-Kelley, A. Banerjee, J.-Q. Yan, C. A. Bridges, D. G. Mandrus, S. E. Nagler, A. K. Kolezhuk, and S. A. Zvyagin, Unconventional spin dynamics in the honeycomb-lattice material $\alpha-\mathrm{RuCl}_{3}$ : High-field electron spin resonance studies, Phys. Rev. B 96, 241107(R) (2017).

[65] H. B. Cao, A. Banerjee, J.-Q. Yan, C. A. Bridges, M. D. Lumsden, D. G. Mandrus, D. A. Tennant, B. C. Chakoumakos, and S. E. Nagler, Low-temperature crystal and magnetic structure of $\alpha-\mathrm{RuCl}_{3}$, Phys. Rev. B 93, 134423 (2016).

[66] S. M. Winter, K. Riedl, D. Kaib, R. Coldea, and R. Valentí, Probing $\alpha-\mathrm{RuCl}_{3}$ Beyond Magnetic Order: Effects of Temperature and Magnetic Field, Phys. Rev. Lett. 120, 077203 (2018).

[67] L. Janssen, E. C. Andrade, and M. Vojta, Honeycomb-Lattice Heisenberg-Kitaev Model in A Magnetic Field: Spin Canting, Metamagnetism, and Vortex Crystals, Phys. Rev. Lett. 117, 277202 (2016).

[68] E. Rastelli, A. Tassi, and L. Reatto, Noncollinear magnetic order and spin wave spectrum in presence of competing exchange interactions, J. Magn. Magn. Mater. 15-18, 357 (1980).

[69] J. B. Fouet, P. Sindzingre, and C. Lhuillier, An investigation of the quantum $\mathrm{J}_{1}-\mathrm{J}_{2}-\mathrm{J}_{3}$ model on the honeycomb lattice, Europhys. J. B 20, 241 (2001).

[70] S. M. Winter, K. Riedl, P. A. Maksimov, A. L. Chernyshev, A. Honecker, and R. Valentí, Breakdown of magnons in a strongly spin-orbital coupled magnet, Nature Commun. 8, 1152 (2017).

[71] J. Greitemann, K. Liu, and L. Pollet, tensorial-kernel SVM library, https://gitlab.physik.uni-muenchen.de/tk-svm/tksvm-op.
[72] B. Schölkopf, A. J. Smola, R. C. Williamson, and P. L. Bartlett, New support vector algorithms, Neural Comput. 12, 1207 (2000).

[73] C.-C. Chang and C.-J. Lin, Training v-support vector classifiers: theory and algorithms, Neural Comput. 13, 2119 (2001).

[74] C.-C. Chang and C.-J. Lin, Libsvm: A library for support vector machines, ACM Trans. Intell. Syst. Technol. 2, 1 (2011).

[75] A. Gaenko, A. E. Antipov, G. Carcassi, T. Chen, X. Chen, Q. Dong, L. Gamper, J. Gukelberger, R. Igarashi, S. Iskakov, M. Könz, J. P. F. LeBlanc, R. Levy, P. N. Ma, J. E. Paki, H. Shinaoka, S. Todo, M. Troyer, and E. Gull, Updated core libraries of the ALPS project, Comput. Phys. Commun. 213, 235 (2017).

[76] J. Greitemann, Investigation of hidden multipolar spin order in frustrated magnets using interpretable machine learning techniques, Ludwig Maximilians University at München, 2019.

[77] K. Liu, J. Nissinen, R.-J. Slager, K. Wu, and J. Zaanen, Generalized Liquid Crystals: Giant Fluctuations and the Vestigial Chiral Order of $I, O$, and $T$ Matter, Phys. Rev. X 6, 041025 (2016).

[78] J. Nissinen, K. Liu, R.-J. Slager, K. Wu, and J. Zaanen, Classification of point-group-symmetric orientational ordering tensors, Phys. Rev. E 94, 022701 (2016).

[79] L. Michel, Symmetry, invariants, topology. Basic tools, Phys. Rep. 341, 11 (2001).

[80] C. Miles, A. Bohrdt, R. Wu, C. Chiu, M. Xu, G. Ji, M. Greiner, K. Q. Weinberger, E. Demler, and E.-A. Kim, Correlator convolutional neural networks as an interpretable architecture for image-like quantum matter data, Nature Commun. 12, 3905 (2021).

[81] C.-W. Hsu and C.-J. Lin, A comparison of methods for multiclass support vector machines, IEEE transactions on Neural Networks 13, 732 (2002).

[82] V. N. Vapnik, Statistical Learning Theory (Wiley, New York, 1998).

[83] N. Rao, K. Liu, and L. Pollet, Inferring hidden symmetries of exotic magnets from detecting explicit order parameters, Phys. Rev. E 104, 015311 (2021).

[84] M. Fiedler, Algebraic connectivity of graphs, Czech. Math. J. 23, 298 (1973).

[85] M. Fiedler, A property of eigenvectors of nonnegative symmetric matrices and its application to graph theory, Czech. Math. J. 25, 619 (1975).

[86] H. G. Katzgraber, S. Trebst, D. A. Huse, and M. Troyer, Feedback-optimized parallel tempering monte carlo, J. Stat. Mech.: Theory Exp. (2006) P03018. 